\title{
Síntesis y caracterización de fotocatalizadores de hierro soportado sobre zeolitas microporosas
}

\author{
Synthesis and characterization of iron photocatalysts \\ supported on microporous zeolites
}

Laura Lerici, Carla Femanelli, Eliana Diguilio, Liliana Pierella, Clara Saux

\author{
CITeQ (Centro de Investigación y Tecnología Química), CONICET UTN-FRC, Av. Cruz Roja Argentina esq. Maestro \\ López. Ciudad Universitaria, Córdoba, Argentina \\ e-mail:Correo electrónico de contacto: 1lerici@frc.utn.edu.ar
}

\begin{abstract}
RESUMEN
Se sintetizaron materiales zeolíticos del tipo ZSM11 y BETA por el método hidrotérmico, impregnados con $6 \%$ de hierro y se caracterizaron por diversas técnicas fisicoquímicas, para ser empleados en la degradación fotocatalítica del Naranja de Metilo (MO). Mediante difracción de rayos X (XRD) y Análisis Infrarrojo con Transformada de Fourier (FTIR) se verificó la estructura y cristalinidad de las matrices y sus expresiones modificadas. Los patrones de difracción mostraron las señales características a $2 \theta=7,9,23$ y $24^{\circ}$, para la matriz ZSM11, y $2 \theta=7-8^{\circ}$ y $21-22^{\circ}$, para la BETA. El área superficial determinada por BET fue de $392 \mathrm{~m}^{2} / \mathrm{g}$ para la ZSM11 y $585 \mathrm{~m}^{2} / \mathrm{g}$ para la BETA. En ambos casos se produjo una disminución del área superficial con la incorporación del metal. El contenido de hierro fue confirmado por Absorción Atómica, obteniéndose porcentajes similares a los teóricos. Además, por SEM y EDX, se detectaron señales a 0,5 y 6,5 KeV, correspondientes a la presencia de hierro en la matriz zeolítica. Por Espectroscopia de UVvis con Reflectancia Difusa (DRS-UVvis) se calcularon las energías de band gap de los materiales, obteniéndose resultados similares al $\alpha-\mathrm{Fe}_{2} \mathrm{O}_{3}$. Mediante esta técnica se verificó la disminución de las energías de band gap con respecto a la matriz zeolítica correspondiente. Los materiales fueron evaluados catalíticamente en la fotodegradación del MO obteniendo porcentajes de degradación de 96 \% para la Fe-ZSM11 y $56 \%$ para la Fe-BETA.
\end{abstract}

Palabras clave: Fe-ZSM11, Fe-BETA, Naranja de Metilo, Degradación Fotocatalítica.

\begin{abstract}
ZSM11 and BETA zeolitic materials were synthesized by the hydrothermal method and were further impregnated with a $6 \mathrm{wt} \%$ of iron and characterized by different physicochemical techniques in order to be employed in the photocatalytic degradation of methyl orange (MO). Matrices structures and crystallinity were verified by X Ray Diffraction (XRD) and Fourier Transformed Infrared Spectroscopy (FTIR), even for modified zeolites. XRD patterns showed the characteristic signals at $2 \theta=7,9,23$ and $24^{\circ}$ for ZSM11 matrix, and $2 \theta=7-8^{\circ}$ and $21-22^{\circ}$, for BETA. According to BET results, specific surface area were $392 \mathrm{~m}^{2} / \mathrm{g}$ for ZSM11 and $585 \mathrm{~m}^{2} / \mathrm{g}$ for BETA. In both cases, an area reduction with metal incorporation were obtained. Iron content was confirmed by Atomic Absorption (AA) spectroscopy, finding similar values than the theoretical ones. Additionaly, iron signals $(0,5$ y $6,5 \mathrm{KeV})$ were detected by SEM EDX. Band gap energies of the materials were calculated employing Diffuse Reflectance Spectroscopy- UVvis (DRS-UVvis), obtaining similar values than $\alpha-\mathrm{Fe}_{2} \mathrm{O}_{3}$. A reduction of the band gap energy in comparison with the correspondent material was also found by this technique. The materials were further evaluated as catalysts for MO photodegradation obtaining a $96 \%$ and $56 \%$ of degradation for Fe-ZSM11 and Fe-BETA, respectively.
\end{abstract}

Keywords: Fe-ZSM11, Fe-BETA, Methyl Orange, Photocatalytic Degradation.

\section{INTRODUCCIÓN}

Un grupo importante de contaminantes orgánicos en agua son los colorantes. Su principal aplicación es el 
teñido de fibras textiles, cuero, papel y pinturas y la fabricación de tintas para impresión. La descarga de efluentes coloreados a los cauces de los ríos interfiere con la difusión de la luz solar dificultando la fotosíntesis y el crecimiento de la vida acuática. Además, los colorantes disminuyen los niveles de oxígeno y confieren alta toxicidad al agua pudiendo ser cancerígenos y mutagénicos [1]. En general, las moléculas de los colorantes utilizados en la actualidad son de estructuras muy variadas y complejas. La mayoría de ellos son de origen sintético, muy solubles en agua, altamente resistentes a la acción de agentes químicos y poco biodegradables. El Naranja de Metilo es un colorante azoico que presenta gran estabilidad y toxicidad y es resistente a los tratamientos convencionales [2]. Su toxicidad y persistencia afecta directamente la salud de los ecosistemas y representa una amenaza para los seres humanos al contaminar los suministros de agua potable, tales como aguas superficiales y subterráneas. Los sistemas de oxidación fotocatalítica se presentan como una alternativa eficaz para la purificación de aguas contaminadas empleando como fuente energética luz UVVis.

En particular, la fotocatálisis heterogénea, a diferencia de la mayoría de los procesos fotoquímicos, puede emplearse para tratar mezclas complejas de contaminantes con la posibilidad de utilización de la radiación solar como fuente primaria de energía [3,4]. Las especies fotoquímicas empleadas son semiconductores que mediante la incidencia de un haz de luz con energía suficiente, son capaces de generar agentes óxidoreductores con suficiente vida media y reactividad para transformar a los contaminantes (inorgánicos y orgánicos) en productos de menor riesgo medioambiental. Existen diversos materiales con propiedades adecuadas para actuar como fotocatalizadores, tales como los óxidos de titanio $\left(\mathrm{TiO}_{2}\right)$, hierro $\left(\mathrm{Fe}_{2} \mathrm{O}_{3}\right)$, estaño $\left(\mathrm{SnO}_{2}\right)$, zinc $(\mathrm{ZnO})$, y sulfuros de zinc $(\mathrm{ZnS})$ y de cadmio $(\mathrm{CdS})$, entre otros. La búsqueda continua de mejoras para los sistemas fotocatalíticos heterogéneos ha llevado al uso de semiconductores soportados con el fin de incrementar el área específica y así la actividad por sitio activo, además de evitar el fenómeno de sinterización. Entre los soportes comunes reportados en bibliografía, podemos mencionar la $\mathrm{SiO}_{2}$ [5], $\mathrm{Al}_{2} \mathrm{O}_{3}$ [6], fibras de vidrio [7], zeolitas [8,9], entre otros. En particular las zeolitas son materiales cristalinos inorgánicos y microporosos capaces de adsorber moléculas orgánicas. A pesar de no poseer actividad fotocatalítica por sí mismas, ellas pueden actuar como soportes gracias a la capacidad de anclar especies fotocatalíticas en su superficie.

El uso de zeolitas como soporte proporciona propiedades físicas fotoespecíficas, tales como control de la transferencia de carga y de los procesos de intercambio de electrones. Las zeolitas del tipo A, H-ZSM-5, $\mathrm{H}-\mathrm{Y}$ y H-BETA, entre otras, demostraron ser altamente efectivas como soporte para el $\mathrm{TiO}_{2}$, mejorando la actividad fotocatalítica de los catalizadores obtenidos respecto de la actividad del $\mathrm{TiO}_{2}$ utilizado sin soportar $[10,11]$.

El presente trabajo tiene el objetivo de sintetizar y caracterizar materiales catalíticos para ser empleados en la eliminación fotocatalítica del Naranja de Metilo presente en los cursos de agua.

\section{MATERIALES Y MÉTODOS}

Las matrices zeolíticas se obtuvieron por síntesis hidrotérmica utilizando como agente plantilla hidróxido de tetrabutilamonio, en el caso de la ZSM11, e hidróxido de tetraetilamonio, para la BETA. Luego se desorbieron bajo atmósfera de $\mathrm{N}_{2}$, con un flujo de $20 \mathrm{~mL} / \mathrm{min}$, desde $110{ }^{\circ} \mathrm{C}$ a $500{ }^{\circ} \mathrm{C}$, a temperatura programada $(10$ ${ }^{\circ} \mathrm{C} / \mathrm{min}$ ) y se calcinaron en atmósfera estática y oxidante (mufla) a $500{ }^{\circ} \mathrm{C}$ durante $12 \mathrm{~h}$ para obtener las expresiones sódica de las zeolitas. Las formas amonio de los catalizadores $\left(\mathrm{NH}_{4}\right.$-Zeolita) se prepararon por intercambio iónico con una solución de cloruro de amonio $1 \mathrm{M}$ a $80{ }^{\circ} \mathrm{C}$ durante $40 \mathrm{~h}$. La incorporación de hierro se realizó por impregnación con sulfato de hierro (II) heptahidratado $\left(\mathrm{FeSO}_{4} \cdot 7 \mathrm{H}_{2} \mathrm{O}\right)$. Luego se desorbieron bajo atmósfera de $\mathrm{N}_{2}$ a $500{ }^{\circ} \mathrm{C}$ y se calcinaron a $500{ }^{\circ} \mathrm{C}$ por $8 \mathrm{~h}$.

Los catalizadores fueron caracterizados en un difractómetro de rayos X (XRD) marca PANalyticalX'pert PRO, usando radiación monocromática de $\mathrm{CuK} \alpha$ de $1,54 \AA$ longitud de onda, barriendo desde 5 a $50^{\circ}(2 \theta)$ con un ancho de paso de 0,2 grados. La determinación del área superficial se realizó por el método BET utilizando un instrumento Micromeritics Pulse Chemisorb 2700 por adsorción de $\mathrm{N}_{2}$ a $77 \mathrm{~K}$. La cantidad de hierro impregnado se determinó por absorción atómica en un espectrómetro Perkin Elmer 3100. El análisis de espectroscopia infrarrojo con transformada de Fourier (FTIR) se realizó en un espectrómetro JASCO 5300. A los fines de confirmar estructura por FTIR se prepararon pastillas al 0,05\% de zeolita en $\mathrm{KBr}$, efectuando el análisis en la región de vibración de la red zeolítica (FTIR de fondo), entre $400-1800 \mathrm{~cm}^{-1}$. La espectroscopia de UV-Vis con reflectancia difusa (DRS-UVvis) permitió el análisis del entorno de coordinación y la posición del hierro, en un espectrofotómetro JASCO V 650 equipado con esfera integradora.

Los ensayos de fotodegradación se llevaron a cabo en un reactor cilíndrico de vidrio Pirex provisto de una camisa de refrigeración con circulación de agua para mantener la temperatura a $25-30{ }^{\circ} \mathrm{C}$. El reactor fue irradiado por una lámpara de mercurio de alta presión de $125 \mathrm{~W}$ (con un máximo de emisión alrededor de 
$365 \mathrm{~nm}$ ) que se ubicó en el interior de la camisa refrigerante y se sumergió en la solución del colorante. El catalizador se mantuvo en suspensión por medio de agitación magnética y se burbujeó con aire en forma continua durante todo el ensayo para favorecer la suspensión del catalizador y asegurar altos niveles de oxígeno en el sistema de reacción. Se retiraron alícuotas a determinados intervalos de tiempo de reacción. Las mismas fueron filtradas y analizadas en un espectrómetro UV a $464 \mathrm{~nm}$. El tiempo total de reacción fue de 240 minutos y la concentración inicial de MO y del catalizador fue de $8 \times 10^{-5} \mathrm{~mol} / \mathrm{L}$ y $500 \mathrm{mg} / \mathrm{L}$, respectivamente. El porcentaje de degradación del MO fue calculado como:

Degradación $\%=(1-\mathrm{C} / \mathrm{C} 0) * 100$

donde C0 concentración inicial de MO y C la concentración de MO al tiempo t.

\section{RESULTADOS}

La zeolita Fe-ZSM11 y Fe-BETA impregnadas al 6\% de hierro fueron caracterizadas por diversas técnicas analíticas. Por XRD (Figura 1), se observaron las señales características a $2 \theta=7$ y 9 grados y $2 \theta=23$ y 24 grados para las zeolitas ZSM11 y $2 \theta=7-8$ y $2 \theta=21$ y 22 grados para las zeolitas BETA [12]. En el caso de la muestra Fe-BETA es posible distinguir las señales a 33.2 y $35.5^{\circ}$, correspondientes a la presencia de $\alpha-\mathrm{Fe}_{2} \mathrm{O}_{3}$ [13]. Tanto por XRD como por FTIR en la zona de huella dactilar, se confirmó que la estructura y cristalinidad se conservaron después de los distintos tratamientos químicos y térmicos realizados.
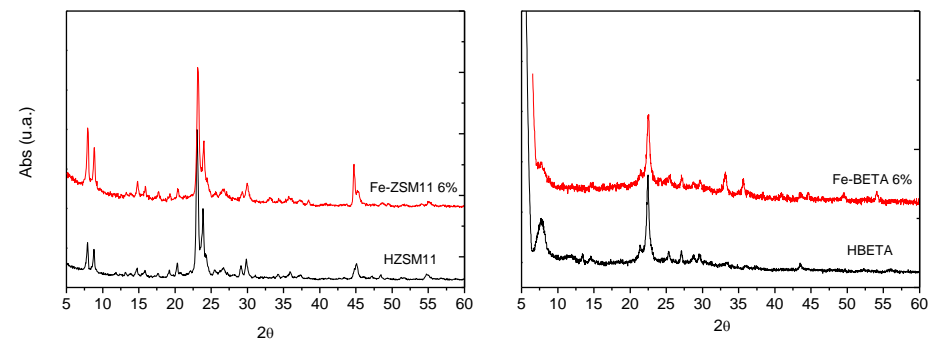

Figura 1: Patrones de difracción de las zeolitas ZSM11 y BETA y sus expresiones modificadas con hierro.

Como puede observarse en la Tabla 1, la incorporación del catión $\mathrm{Fe}^{3+}$ generó una disminución del área superficial de las zeolitas (efecto atribuído a la agregación de los metales sobre la superficie lo que produce el bloqueo de los poros de la matriz [14]).

Tabla 1: Caracterización físico-química de los catalizadores.

\begin{tabular}{|c|c|c|}
\hline ZEOLITA & $\mathbf{S}_{\text {BET }}\left(\mathbf{m}^{\mathbf{2}} / \mathbf{g}\right)$ & $\mathbf{F e}(\% \mathbf{p} / \mathbf{p})$ \\
\hline H-ZSM11 & 392 & - \\
\hline Fe-ZSM11 & 318 & 4,43 \\
\hline H-BETA & 585 & - \\
\hline Fe-BETA & 463 & 5,75 \\
\hline
\end{tabular}

El entorno de coordinación de los metales de transición se estudió por DRS UV-Vis. Analizando los espectros correspondientes a las muestras Fe-ZSM11 y Fe-BETA (Figura 2), se observan bandas de absorción significativas para ambas zeolitas lo que favorece la generación de mayor cantidad de pares huecoelectrón, responsables de promover las reacciones fotocatalíticas [15]. Se pueden diferenciar tres regiones en el rango de 200-300, 300-450 y 450-650 nm. La primera asociada a la transferencia de carga $\left(\mathrm{O} \rightarrow \mathrm{Fe}^{3+}\right)$ entre el hierro y el oxígeno en donde indica que el $\mathrm{Fe}^{3+}$ pudo incorporarse como cationes aislados. Los picos a longitudes de onda $<250 \mathrm{~nm}$ y entre $250-300 \mathrm{~nm}$ aproximadamente, corresponden a la presencia de iones aislados $\mathrm{Fe}^{3+}$ en coordinación tetraédrica y octaédrica, respectivamente [16].

Las energías de band gap de los materiales catalíticos fueron determinadas usando la función de remi- 
sión de Kubelka-Munk. Los valores de band gap para ambos materiales fueron cercanos al reportado en bibliografía para el $\alpha-\mathrm{Fe}_{2} \mathrm{O}_{3}(2,2 \mathrm{eV})$ [17], con valores de $2 \mathrm{eV}$ y 1,9 eV para la Fe-ZSM11 y Fe-BETA, respectivamente. Este resultado es acorde con lo reportado por M. Noorjahan et al. [18] que encuentran que con el aumento del contenido de hierro soportado sobre zeolitas Y, los valores de Eg se acercan al del óxido puro. Cabe destacar que los autores reportan un valor máximo de incorporación del metal del $5 \%$ p/p, siendo el contenido de Fe en las muestras presentadas en este trabajo superior. Este comportamiento se debería al incremento en el número de los niveles de energía que provocan una reducción del band gap.

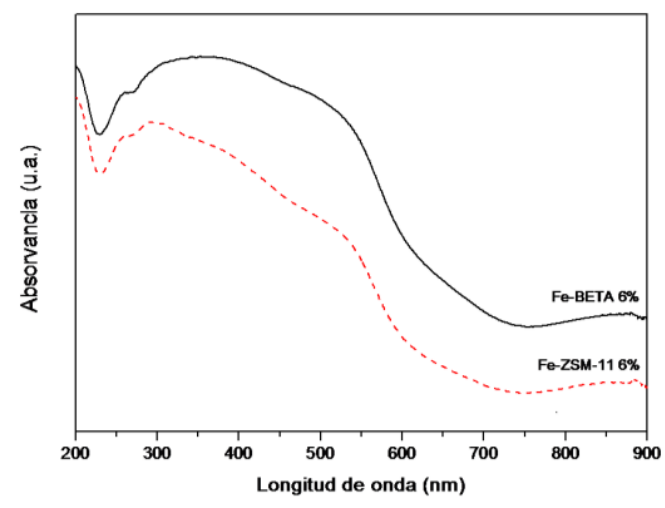

Figura 2: Espectro DRS UV-Vis de las Fe-Zeolitas a 6\%.

La presencia de hierro en las matrices zeolíticas fue confirmada por los espectros SEM-EDX por la aparición de las señales a 0,5 y 6,4 keV aproximadamente, correspondientes a las radiaciones $\mathrm{Fe} \mathrm{L} \alpha 1$ y O K $\alpha$, y K $\alpha$ Fe, respectivamente (Figura 3) [19]. Además, por esta técnica se verificó la presencia de silicio, aluminio y oxígeno.
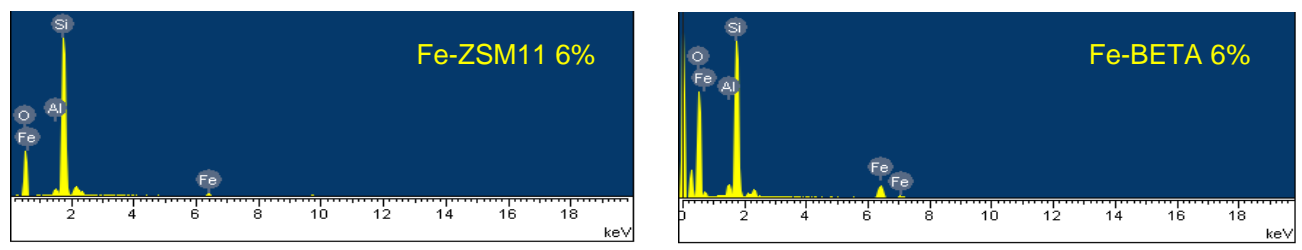

Figura 3: Espectros SEM-EDX de las Fe-Zeolitas

Se efectuaron dos experiencias de control previas a los ensayos fotocatalíticos con el objetivo de evaluar la actividad de ambos catalizadores. En primer lugar, se llevó a cabo una fotólisis (exponiendo la solución de MO a la radiación UV en ausencia de catalizador) y en segundo lugar se realizó un ensayo fotocatalítico con $\mathrm{Fe}_{2} \mathrm{O}_{3}$ comercial. Ambas experiencias mostraron un efecto insignificante en la degradación del colorante con porcentajes de degradación del MO inferior al $1 \%$ en el caso de la fotólisis y del $4 \%$ para la experiencia con el óxido de hierro. Las experiencias fotocatalíticas, con una concentración de Fe-Zeolita de 500 $\mathrm{mg} / \mathrm{L}$, revelaron porcentajes de degradación de MO del 96 y $56 \%$ para la Fe-ZSM11 y Fe-BETA, respectivamente (Figura 4), lo que permite corroborar una mejora en la eliminación del MO debida al agregado del fotocatalizador de hierro soportado sobre las matrices zeolíticas. Todos los ensayos se diseñaron de manera de mantener los 30 primeros minutos en la oscuridad para favorecer la adsorción del colorante sobre los materiales catalíticos. Es necesario aclarar que los porcentajes de degradación reportados presentan una contribución debida a la adsorción del colorante y otra debida al proceso de degradación fotocatalítico propiamente dicho. En el caso de la Fe-ZSM11, se puede observar una adsorción inicial importante del MO sobre la superficie del catalizador ( 80 \%), ascendiendo a sólo el 11\% para la Fe-BETA. En este punto es importante remarcar que los resultados tan dispares obtenidos al catalizar la fotodegradación con hierro soportado se deben, por una parte, al efecto promotor de la matriz zeolítica que favorece la retención de las moléculas del contaminante por sus características inherentes. Por otro lado, al aumento considerable del área superficial expuesta de las especies de Fe presentes, con respecto al óxido másico (Cabe aclarar que se empleó en todas las reacciones catalíticas idénticas cantidades de moles del metal). El anclaje del Fe sobre estos aluminosili- 
catos microporosos impide el aglomerado de partículas, asegurando una mayor dispersión de las especies activas y favoreciendo su comportamiento como fotocalizador.

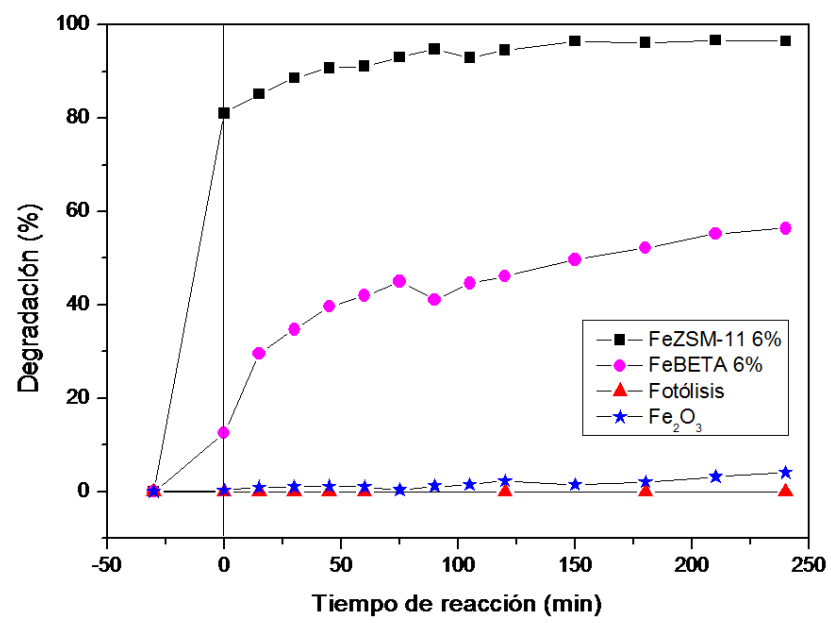

Figura 4: Degradación catalítica del MO.

\section{CONCLUSIONES}

Los resultados reportados demuestran que es posible la síntesis y funcionalización de las zeolitas ZSM11 y BETA para su uso en fotocatálisis. La incorporación de las especies fotocatalíticas activas sobre las matrices de las zeolitas, no generó pérdida de estructura ni cristalinidad de los materiales. Por AA, DRS UV-Vis y SEM-EDX se pudieron verificar y cuantificar la presencia de las especies hierro sobre las ZSM11 y BETA, obteniéndose materiales con valores de band gap similares a los reportados para los semiconductores más estudiados $\left(\mathrm{TiO}_{2}, \mathrm{CdS}, \mathrm{ZnO}\right.$, entre otros), lo que permite un mayor aprovechamiento del espectro de emisión de la lámpara. Los ensayos catalíticos demostraron porcentajes de eliminación del MO de 96 y $56 \%$. Superando ampliamente los valores obtenidos al emplear $\mathrm{Fe}_{2} \mathrm{O}_{3}$ comercial gracias al efecto promotor del soporte empleado que favoreció la retención del contaminante y evitó la sinterización con pérdida de superficie activa expuesta. Por este motivo, es posible considerar a las zeolitas modificadas con hierro como prometedores fotocatalizadores.

\section{AGRADECIMIENTOS}

Este trabajo fue parcialmente financiado por el Programa de Incentivos a Docentes Investigadores (20142017) UTN 2099. Agradecemos a CONICET: L.B. Pierella y C. Saux (investigadores) y L. Lerici, C. Fermanelli y E. Diguilio (becarias).

\section{BIBLIOGRAFÍA}

[1] LACHHEB, H., PUZENAT, E., HOUAS, A., et al., "Photocatalytic degradation of various types of dyes (Alizarin S, Crocein Orange G, Methyl Red, Congo Red, Methylene Blue) in water by UV-irradiated titania" Applied Catalysis B: Environmental, v. 39, n. 1, pp. 75-90, 2002.

[2] CHEN, Y. P., LIU, S.-Y., YU, H.-Q., et al., "Radiation-induced degradation of methyl orange in aqueous solutions" Chemosphere, v. 72, n. 4, pp. 532-536, 2008.

[3] ANDRADE, F. V., LIMA, G.M., AUGUSTI, R., et al., "A novel $\mathrm{TiO}_{2} /$ autoclaved cellular concrete composite: From a precast building material to a new floating photocatalyst for degradation of organic water contaminants", Journal of Water Process Engineering., v. 7, pp. 27-35, 2015.

[4] SIVAGAMI, K., KRISHNA, R.R., SWAMINATHAN, T. "Photo catalytic degradation of pesticides in immobilized bead photo reactor under solar irradiation", Solar Energy, v. 103, pp. 488-493, 2014.

[5] WANG, H.L., LIANG, W.Z., JIANG, W.F. "Solar photocatalytic degradation of 2-sec-butyl-4,6dinitrophenol (DNBP) using $\mathrm{TiO}_{2} / \mathrm{SiO}_{2}$ aerogel composite photocatalysts", Materials Chemistry and Physics. v.130, pp. 1372-1379, 2011. 
[6] LI, Z., SHENG, J., WANG Y., et al., "Enhanced photocatalytic activity and stability of alumina supported hematite for azo-dye degradation in aerated Y aqueous suspension”, Journal of Hazardous Materials., v. 254-255, pp. 18-25, 2013.

[7] ERJAVEC, B., HUDOKLIN, P., PERC, K., et al., "Glass fiber-supported $\mathrm{TiO}_{2}$ photocatalyst: Efficient mineralization and removal of toxicity/estrogenicity of bisphenol A and its analogs", Applied Catalysis B, Environmental., v. 183, pp. 149-158, 2016.

[8] KUWAHARA, Y., AOYAMA, J., MIYAKUBO, K., et al., " $\mathrm{TiO}_{2}$ photocatalyst for degradation of organic compounds in water and air supported on highly hydrophobic FAU zeolite: Structural, sorptive, and photocatalytic studies", Journal of Catalysis, v. 285, pp. 223-234, 2012.

[9] PAN, Z., STEMMLER, E.A., CHO, H.J., et al., "Photocatalytic degradation of $17 \alpha$-ethinylestradiol (EE2) in the presence of $\mathrm{TiO}_{2}$-doped zeolite", Journal of Hazardous Materials, v. 279, pp. 17-25, 2014

[10] MOHAMED, R.M., ISMAIL, A.A., OTHMAN, I., ET AL., "Preparation of $\mathrm{TiO}_{2}-\mathrm{ZSM}-5$ zeolite for photodegradation of EDTA”, Journal of Molecular Catalysis A: Chemical, v. 238, pp. 151-157, 2005.

[11] SHANKAR, M. V., CHERALATHAN, K.K., ARABINDOO, B., et al., "Enhanced photocatalytic activity for the destruction of monocrotophos pesticide by $\mathrm{TiO}_{2} / \mathrm{H} \beta$ ", Journal of Molecular Catalysis A: Chemical. v. 223, pp. 195-200, 2004.

[12] RENZINI, M. S., LERICI, L. C., SEDRAN, U., et al., "Stability of ZSM-11 and BETA zeolites during the catalytic cracking of low-density polyethylene", Journal of Analytical and Applied Pyrolysis, v. 92, n. 2, pp. 450-455, Nov. 2011.

[13] QI, G., YANG, R. T. "Selective catalytic oxidation (SCO) of ammonia to nitrogen over Fe/ZSM-5 catalysts", Applied Catalysis A: General, v. 287, n. 1, pp. 25-33, 2005.

[14] REGENHARDT, S. A., MEYER, C. I., TRASARTI, A. F., et al., "Catalytic oxidation of carbon tetrachloride on metal exchanged Y-zeolite”, Chemical Engineering Journal, v. 198-199, pp. 18-26, Aug. 2012.

[15] WANG, C., HUANG, Z. "Controlled synthesis of $\alpha-\mathrm{Fe} 2 \mathrm{O} 3$ nanostructures for efficient photocatalysis", Materials Letters, v. 164, pp. 194-197, 2016.

[16] LU, Y., ZHENG, J., LIU, J., et al., "Fe-containing mesoporous silicates with macro-lamellar morphology”, Microporous Mesoporous Materials, v. 106, n. 1-3, pp. 28-34, 2007.

[17] JAAFAR, N. F., ABDUL JALIL, A., TRIWAHYONO, S., et al., "Photodecolorization of methyl orange over $\alpha$-Fe $\mathrm{O}_{3}$-supported HY catalysts: The effects of catalyst preparation and dealumination", Chemical Engineering Journal, v. 191, pp. 112-122, 2012.

[18] NOORJAHAN, M., DURGA KUMARI, V., SUBRAHMANYAM, M., et al., "An efficient and stable photo-Fenton catalyst” Applied Catalysis B, Environmental, v. 57, pp. 291-298, 2005.

[19] MOHAN, N., CINDRELLA, L. "Direct synthesis of Fe-ZSM-5 zeolite and its prospects as efficient electrode material in methanol fuel cell”, Materials Science in Semiconductor Processing, v. 40, pp. 361-368, 2015. 